\title{
Effects of decision criterion on response latencies of binary decisions
}

\author{
BLAS ESPINOZA-VARAS and CHARLES S. WATSON \\ Central Institute for the Deaf, St. Louis, Missouri
}

\begin{abstract}
The effects of decision criterion on response latencies of binary decisions were examined. The stimuli comprised two, partly overlapping, "normal" distributions of either two-digit numbers or tonal frequencies. Individual stimuli were randomly sampled from the distributions, and subjects had to decide from which distribution the stimulus was sampled. The decision goal was to maximize the expected gain using three different payoff matrices. Decision latencies with and without prior knowledge of optimal decision criteria were measured. In a control task, subjects were asked only to label stimuli as being either above or below the optimal criteria (without regard for a posteriori probabilities or expected gain). The relation between criterion and latency was examined by means of a trial-by-trial analysis of the stimuli and the responses. Response latency was inversely related both to the distance between the stimulus and the decision criterion and to the probability of the response elicited by the stimulus. Response latencies showed a maximum at the criterion in all conditions. These results were obtained with different stimulus modalities (tones or numbers), different discriminability levels within the auditory modality, and different decision tasks. It is proposed that the decision criterion is the primary determinant of response latencies of binary decisions.
\end{abstract}

Several authors have proposed that analyses of response times ${ }^{1}$ in signal-detection experiments could help in the understanding of processes underlying sensory decisions (e.g., Carterette, Friedman, \& Cosmides, 1965; Emmerich, Gray, Watson, \& Tanis, 1972; Green \& Swets, 1966; Pike, 1973; Ratcliff, 1985, 1987). Much of this work has been guided by the hypothesis that response latency is a function of the distance between the stimulus and the observer's decision criterion (e.g., Emmerich et al., 1972; Gescheider, Wright, \& Evans, 1968; Gescheider, Wright, Weber, Kirchner, \& Milligan, 1969; Norman \& Wickelgren, 1969; see Ratcliff, 1985, 1987, for a recent review). The hypothesis follows from the assumption of signaldetection theory that sensory observations are scaled in terms of likelihood ratios, $l_{\mathrm{x}}$, and that decisions are made

This research was supported by NIH Grant 03856 to the Central Institute for the Deaf. B. Espinoza-Varas was supported by a FulbrightHays Grant. Preparation of this manuscript was supported by grants from the Air Force Office of Scientific Research, the National Institutes of Health to Indiana University, the Oklahoma Center for the Advancement of Science and Technology, and the Presbyterian Health Foundation to the University of Oklahoma Health Sciences Center. Special thanks to Patricia K. Kuhl, William M. Fisher, Brian L. Scott, and Charles K. Burdick, who participated as subjects in Experiment 2. The editorial comments of an anonymous reviewer, Roger Ratcliff, and Neal Macmillan are greatly appreciated. Christi E. Burgin performed the statistical analysis of the data. C. S. Watson is a member of the Department of Speech and Hearing Sciences at Indiana University in Bloomington. Correspondence should be addressed to B. Espinoza-Varas, Department of Communication Sciences and Disorders, University of Oklahoma Health Sciences Center, 825 NE 14th St., Oklahoma City, OK 73190.

-Accepted by previous editor, Charles W. Eriksen by comparing the likelihood ratio associated with each observation, $l x_{\mathrm{o}}$, to a criterion likelihood ratio, $l x_{\mathrm{c}}$. Thus, decision difficulty is assumed to be maximal for observations falling near the criterion (i.e., for small distances between $l x_{\mathrm{o}}$ and $l x_{\mathrm{c}}$ ), and decision latency should follow the well-known direct relation with difficulty.

Indirect evidence supporting the hypothesis was reported by Gescheider et al. (1968), Gescheider et al. (1969), and Emmerich et al. (1972). In experiments on detectability of vibrotactile signals, Gescheider and colleagues varied the a priori probability and the level of the signals to bias the response probabilities. They found that reaction times of responses with low probability (e.g., "signal" responses made under a strict criterion) were longer than those of high-probability responses. This result would support the hypothesis because, on average, sensory inputs eliciting low-probability responses are closer to the criterion than are those eliciting high-probability responses. Emmerich et al. (1972) extended this conclusion to the case of detection of auditory signals. Criterion effects on response latency have been obtained with changes in the a priori probability of the signals (Carterette et al., 1965; Gescheider et al., 1969), in the payoff matrices, in the instructions (Emmerich et al., 1972), and in the signal-to-noise ratio (Gescheider et al., 1969). Another result that supports the hypothesis is that, for a given stimulus alternative, the average latency of incorrect responses is longer than the average latency of correct responses (Carterette et al., 1965; Emmerich et al., 1972, Gescheider et al., 1968; Gescheider et al., 1969). Again, incorrect responses are more likely for sensory inputs near the criterion than for inputs distant from the criterion. 
In spite of the above evidence, the details of the relation between response latency and criterion are not fully understood due in part to shortcomings in the experimental procedures (Ratcliff, 1985, 1987).

First, in the experiments cited, there was no simple direct way of manipulating the variable "distance from criterion," and therefore all available evidence is indirect. For instance, in these experiments, changes in response probabilities are often associated with changes in decision criterion, but the relation between these two variables is not fully understood (e.g., Ratcliff, 1987). In most cases, it is difficult to distinguish between responseprobability and criterion effects on response latency. Second, most of the available evidence derives from detection studies in which the stimulus was degraded and/or the observation time allowed to the subject was limited. Under such conditions, decision and detection aspects of the sensory process are likely to be confounded. Third, as Emmerich et al. (1972) have noted, the available studies have compared average response latencies to average stimuli. For instance, the average latency of the signal responses conditional on signal presentation (hits) is compared to the average latency of another conditional response (e.g., false alarms). However, due to the inherent variability of the stimuli and of the sensory system, some hits are responses to signals that fall close to the decision criterion, while others are responses to signals that fall far from the criterion. The effect of the variable of interest (i.e., distance from criterion) is obscured by this averaging. Finally, most response-time studies have used subjects with limited training in the experimental task; however, most signal-detection studies employ subjects with considerable training in the experimental task.

Clearly, a more detailed description of criterion effects on response latency requires improvement in the experimental procedure. First, the experimental situation should reduce the demands on detection or discrimination processes while preserving the basic features of the signaldetection task. A partial solution is to use stimulus sets in which confusability is determined entirely by the statistics of the stimulus sets, although the individual stimuli within each set can be detected and discriminated without errors (e.g., Kubovy, Rapoport, \& Tversky, 1971; Lee \& Janke, 1964; Ratcliff, 1987). For instance, stimulus sets can be used that are normally distributed and partly overlapping (i.e., confusable), while all individual stimuli within each set are clearly detectable and discriminable from each other. Such stimulus sets would reduce the sensory demands of perceptual decisions, but the demands on the decision process would be similar to those of a standard signal-detection experiment. Achieving the latter condition would be facilitated if the experimental task places no limits on the subjects' observation and response times-that is, if response latencies rather than reaction times are measured to avoid the speed/accuracy tradeoff as much as possible.

Another aspect that requires improvement is the data analysis procedure. The observer's strategy, decision cri- terion, and the distance between the criterion and specific stimuli of the stimulus distributions must be precisely specified. Watson (1962) described a procedure that appears to fulfill these requisites. In experiments on detection of tones masked by Gaussian noise, Watson examined the relation between the subject's confidence ratings and the energy in the stimuli. It was predicted that these two variables should correlate strongly if the observer were able to reproduce the probability densities of the stimuli. The energy in the stimulus waveform was measured on each trial and correlated with the confidence rating on that same trial. The results agreed closely with the prediction, especially because a strong correlation between ratings and stimulus energy was obtained on both noisealone and signal-plus-noise trials. A trial-by-trial analysis of the stimuli allows the specification of the relative location of individual stimuli along the likelihood-ratio axis. A trial-by-trial analysis of the confidence ratings allows the specification of the subject's decision criterion in stimulus units and indicates whether or not the sensory decisions reflect stimulus likelihood ratios.

The aim of the present study was to analyze in detail the relation between criterion and latency in binary decision tasks. The stimuli consisted of normally distributed, partly overlapping stimulus sets, the confusability of which was statistically determined. Individual stimuli within each set could be detected and discriminated with essentially no errors. The relation between latency and criterion was studied directly by means of a trial-by-trial analysis of the stimuli and the responses. Two kinds of stimuli (tone bursts and numbers) were used in conjunction with three different decision tasks that approximated the standard signal-detection situation in different degrees. Highly trained subjects were employed in most of the experiments.

\section{GENERAL METHOD}

\section{Stimulus Distributions}

The stimuli were two sets of either two-digit numbers or tonal frequencies, distributed so as to approximate a normal curve. The stimulus sets had approximately the same standard deviation but different means. Figure 1 shows an outline of the distributions (labeled $A$ and $B$ )

Altogether, the distributions covered a 96-step range with one digit or one frequency interval between steps for numbers or tones, respectively (see description of specific experiments for details of stimulus characteristics). The distributions were generated as follows: the 96-step range was divided into 16 six-step-wide categories or bins. The A distribution covered the 13 lowermost categories, and the B distribution covered the 13 uppermost categories; thus, the distributions shared the 10 middle categories (4th to 13 th). For a given distribution, an element from each of the 13 categories was selected with frequencies of $1,2,3,4,5,6,8,6,5,4,3,2$, and 1 , respectively, yielding a total of 50 stimuli per distribution. Expressed in terms of proportions, these frequencies correspond to $.02, .04, .06, .08, .10, .12, .16, .12, .10, .08, .06, .04$, and .02 , respectively. This procedure allowed presentation of two quasinormal distributions overlapping by $60 \%$ on each block of 100 trials. In addition, the procedure maintained constant within-block stimulus probabilities for subdivisions of the stimulus ranges. The six numerals or tonal frequencies contained in each of the 16 categories 


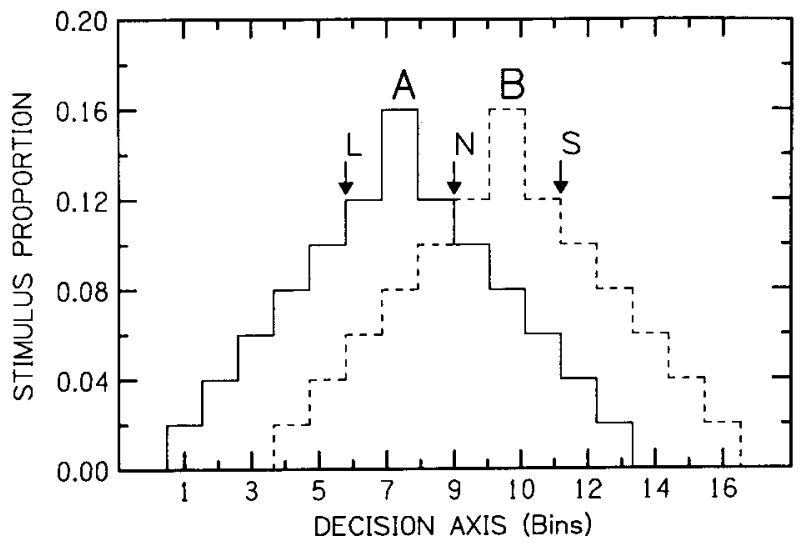

Figure 1. Stimulus distributions $A$ and $B$ used in all the experiments. For each of the $\mathbf{1 6}$ bins of the decision axis, the proportion is shown on the ordinate. Arrows show the locations of the optimal criteria for three payoff matrices: lax (L), neutral (N), and strict (S).

were treated as equivalent in the analysis of the experimental data. Effectively, the decision continuum contained 16 points (see Figure 1). To minimize memory effects, six different randomizations of the A and B distributions were generated. The six randomizations of a given distribution were almost identical in shape, mean, and standard deviation, but they contained different digits or tonal frequencies, depending on the experiment.

\section{Procedure}

On each trial, a stimulus from one of the distributions was randomly selected without replacement. The a priori probability of sampling from the A or the B distribution was .5. The subjects' task was to decide from which distribution the stimulus was sampled. They indicated their decisions on a two-button response box, with the left and the right button corresponding to $A$ and $B$, respectively. The response latencies were measured to the nearest $1.0 \mathrm{msec}$ from the stimulus onset without the subjects' knowledge. The subjects knew the duration of the response interval $(1.75-2.0 \mathrm{sec})$ and were instructed that they would have ample time to make their best decision and that they should be concerned mainly with accuracy, not with how fast they could respond. Any residual concern with speed was minimized by not informing subjects that latencies were being measured. Following the subject's response, feedback lights identified the correct stimulus distribution.

The subjects' decision criteria were varied using three different payoff matrices. Values and costs were chosen with optimal criteria at -0.57 (lax), 0.51 (neutral), and 1.58 (strict) $z$-score units, relative to the mean of the $\mathrm{A}$ distribution (see Figure 1). For the neutral-criterion matrix, the values and costs of each conditional response were $3(\mathrm{a} / \mathrm{A}), 3(\mathrm{~b} / \mathrm{B}),-3(\mathrm{a} / \mathrm{B})$, and $-3(\mathrm{~b} / \mathrm{A})$. For the lax and strict matrices, the corresponding values and costs were $1,4,-3,-2$, and $4,1,-2,-3$.

\section{Decision Tasks}

Three decision tasks were employed: (1) pure statistical decision, (2) instructed statistical decision, and (3) labeling. In the first decision task, the subjects were asked to maximize the overall gain with each payoff matrix and to determine on their own the decisioncriterion values appropriate to each matrix. To further motivate the subjects, a bonus system proportional to the net gain was employed during part of the experiments. The net gain was reported to each subject after each block of trials. The second decision task differed from the first only in that the subjects were told the values of the optimal criteria at the beginning of each block of trials. This cuing was intended to facilitate the use of more stable, close-to-optimal criteria and to reveal whether some of the response latency represents time required to calculate the criterion values. In the third decision task, the subjects were again informed of the optimal criterion values, but they were asked only to label each element of the distributions as larger or smaller (higher or lower for the tonal stimuli) than the optimal criterion. The goal in this task was to maximize the percent correct labeling. ${ }^{2}$ Presumably, the labeling task did not require the subjects to estimate a posteriori probabilities or likelihood ratios nor even to be aware of the existence of the $A$ and $B$ distributions. Two decision tasks were studied in the experiments using number stimuli (pure statistical decision and labeling), whereas all three tasks were employed in the experiments with tones. More specific details of each decision task are given in the sections devoted to the individual experiments.

\section{EXPERIMENT 1}

In the first two experiments, the stimuli were distributions of numbers. The main difference between these two experiments was in the amount of statistical training of the subjects.

\section{Method}

Subjects. The subjects were 3 male high school students, 15.5 years old on the average, with normal vision. They had experience in psychoacoustic experiments, but no formal training in statistics. They were paid for their participation.

Apparatus. The stimuli were two-digit numbers, ranging from 01 to $96(3.0 \times 2.5 \mathrm{~cm}$ per digit), displayed from behind a translucent screen mounted on a sloping box $(32 \times 38 \times 36 \mathrm{~cm})$. The numbers were generated with digitally controlled readouts and could be identified without any difficulty. The response box and the visual display were rigidly attached to the top of a small table so that the subject had to maintain a fixed posture relative to the visual display (approximately $35-40 \mathrm{~cm}$ distance). The subjects worked in individual, sound-treated booths. The presentation of the stimuli, recording of responses, and all other experimental events were controlled by a DEC-PDP $8 / \mathrm{S}$ computer.

Procedure. Two distributions of numbers were generated. The numbers in the A distribution ranged from 01 to 77 , with a mean of 39.5. The B distribution ranged from 19 to 96 , with a mean of 57.5. The standard deviation was approximately 16.8 for both distributions, and the proportions of stimuli above and below a given $z$ score were similar to those of a continuous normal curve. Since the distributions overlapped by about $60 \%$, the theoretical maximum of the signal-detection statistic, $d^{\prime}$, was about 1.1 [i.e., $(57.5-39.5) / 16.8]$.

Each trial included presentation of a randomly sampled number for $265 \mathrm{msec}$ and a 1,750 -msec response interval measured from the stimulus onset. The following trial began $850 \mathrm{msec}$ after the end of the response interval, yielding a 2,600-msec interval between the stimulus onsets of consecutive trials. Pilot experiments with this trial structure showed that subjects were able to identify the numbers with essentially no errors and that after a short period of practice (1-2 h) they could respond comfortably within the response interval. $^{3}$

The subjects were instructed to respond with the left and right thumbs and to maintain, as much as possible, a fixed posture during a block of trials. At the beginning of the experiment, the characteristics of the stimuli, the nature of the decision problem, and the use of payoff matrices were explained to the subjects. Two decision tasks were employed in the experiment: pure statistical decision and labeling. The statistical decision proceeded as described in the previous section. At the beginning of each block of the labeling task, the subjects were given a criterion, or standard, number and 
were asked to decide whether the number presented on each trial was smaller or larger than this standard. ${ }^{4}$ The experimental sessions consisted of 6-8 100-trial blocks separated by rest periods. The experiment included 15 sessions: 4 practice sessions, 8 sessions with pure statistical decision, and 3 sessions with labeling.

\section{Results}

Data analysis. Hit, false-alarm, correct-rejection, and miss proportions, as well as the mean latency of each conditional response were obtained for each of the 16 bins of the stimulus continuum (see Figure 1). Two additional performance measures were obtained: (1) For each of the 16 bins of the decision continuum, the ratio of the total of " $b$ " responses over the total of " $a$ " responses was computed. The stimulus value at which the ratio increased from $\leq 1.0$ to $>1.0$ was defined as the empirical criterion value. (2) The percentage of responses that did not follow the criterion-based decision rule (e.g., correctly responding " $b$ " to a number below the criterion) was defined as the violation rate. ${ }^{5}$ This index provided a measure of criterion stability.

Operating characteristics. Figure 2 (panels labeled A) shows the receiver-operating characteristic (ROC) data obtained on each decision task with three decision criteria. To be consistent with the conventional signal-detection procedure, the ROC data were generated by summation from the right. Thus, the $B$ distribution was defined as the signal plus noise, and the probabilities $\mathrm{P}(\mathrm{b} / \mathrm{B})$ and $\mathrm{P}(\mathrm{b} / \mathrm{A})$ were defined as the hit and false-alarm rates, respectively. The intercept and slope of maximum-likelihood linear fits

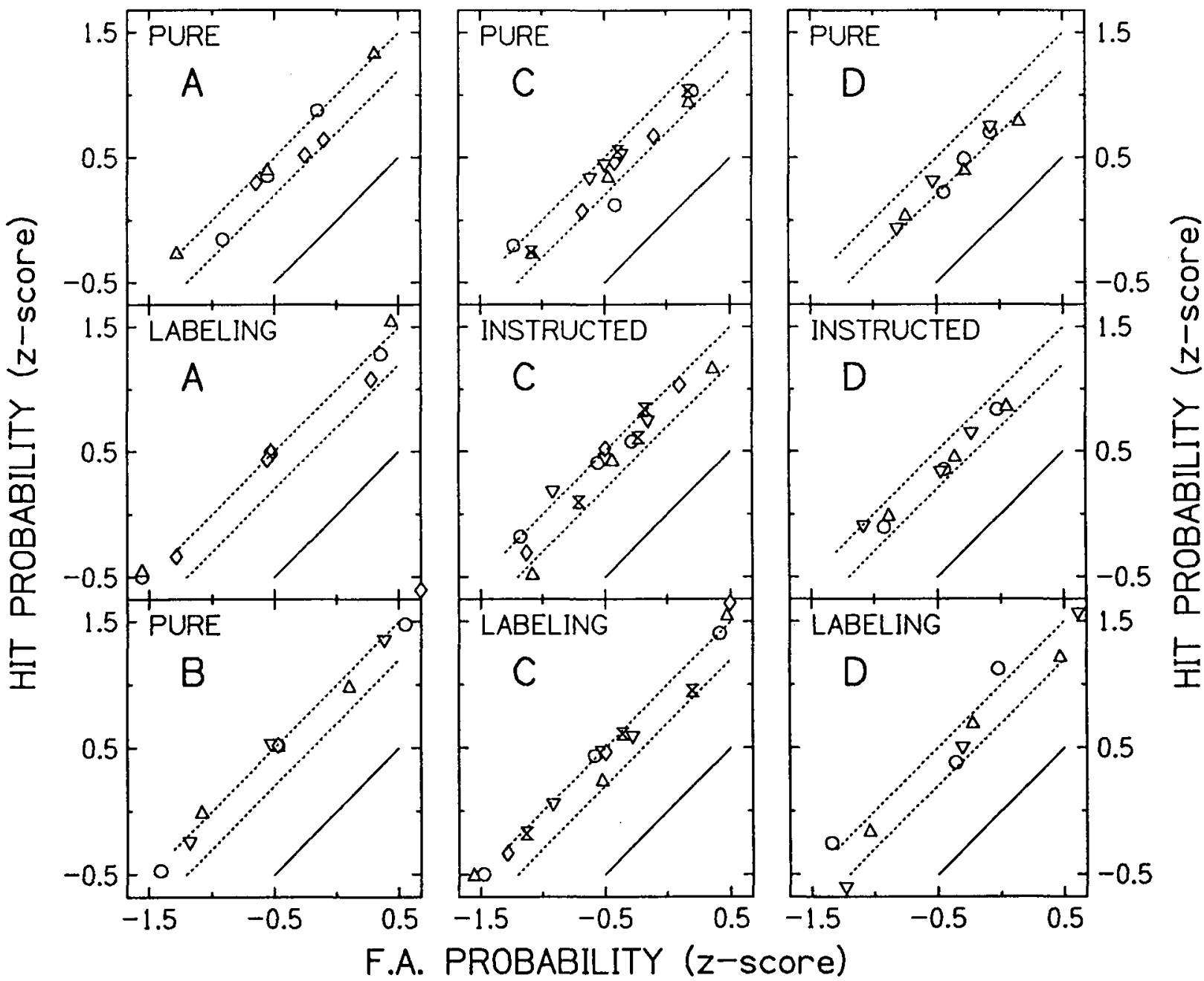

Figure 2. Receiver-operating characteristic (ROC) data obtained in the various experiments. Panels labeled $A$ are for distributions of numbers presented to subjects without training in statistics (Experiment 1 ). The panel labeled B is for distributions of numbers presented to subjects with training in statistics (Experiment 2). Panels labeled $\mathbf{C}$ are for tones with high discriminability (Experiment 3). Panels labeled D are for tones with low discriminability (Experiment 4). The data are represented in normal-probability coordinates. Each panel shows data for a single decision task: pure statistical decision, instructed statistical decision, or labeling. Different symbols within a panel represent different subjects. On each panel, the dotted lines show the theoretical ROC functions yielding $d^{\prime}$ values of 0.7 and 1.0 . Approximately $90 \%$ of the data points fall within this performance range. 
Table 1

Intercept $(a)$ and Slope (b) of Maximum-Likelihood Linear Fits to the Individual ROC Data: (Hit) $=a-b$ (False Alarm), in $z$-score Units

\begin{tabular}{|c|c|c|c|c|c|c|c|}
\hline \multirow[b]{2}{*}{ Experiment } & \multirow[b]{2}{*}{ Task } & \multirow{2}{*}{$\begin{array}{c}\text { Intercept/ } \\
\text { Slope }\end{array}$} & \multicolumn{5}{|c|}{ Subject } \\
\hline & & & 1 & 2 & 3 & 4 & 5 \\
\hline \multirow[t]{3}{*}{1} & Pure & $\begin{array}{l}a \\
b\end{array}$ & $\begin{array}{l}1.1 \\
1.3\end{array}$ & $\begin{array}{l}1.0 \\
0.9\end{array}$ & $\begin{array}{l}0.8 \\
0.8\end{array}$ & & \\
\hline & Labeling & $a$ & 1.0 & 1.1 & 0.9 & & \\
\hline & & $b$ & 0.9 & 1.0 & 0.9 & & \\
\hline \multirow[t]{2}{*}{2} & Pure & $a$ & 1.0 & 0.9 & 1.0 & 1.0 & \\
\hline & & $b$ & 1.0 & 1.0 & 1.0 & 0.9 & \\
\hline \multirow[t]{6}{*}{3} & Pure & $a$ & 0.8 & 0.9 & 0.9 & 1.1 & 0.9 \\
\hline & & $b$ & 0.9 & 1.0 & 0.8 & 1.1 & 1.0 \\
\hline & Instructed & $a$ & 1.0 & 0.9 & 0.9 & 1.0 & 1.1 \\
\hline & & $b$ & 1.0 & 0.9 & 0.8 & 1.0 & 1.1 \\
\hline & Labeling & $a$ & 1.0 & 1.0 & 1.0 & 1.0 & 0.9 \\
\hline & & $b$ & 1.0 & 1.0 & 1.1 & 1.0 & 1.0 \\
\hline \multirow[t]{6}{*}{4} & Pure & $a$ & 1.0 & 0.8 & 0.9 & & \\
\hline & & $b$ & 1.0 & 0.9 & 0.9 & & \\
\hline & Instructed & $a$ & 0.9 & 0.9 & 0.9 & & \\
\hline & & $b$ & 1.0 & 1.0 & 0.9 & & \\
\hline & Labeling & $a$ & 1.0 & 0.9 & 0.9 & & \\
\hline & & $b$ & 1.0 & 0.9 & 1.0 & & \\
\hline
\end{tabular}

Note- $a=d^{\prime}$ value associated with a false-alarm probability of .5 (i.e., $z$ score $=0$ ).

to the ROC data (Dorfman \& Alf, 1968) are given in Table 1.

In both decision tasks, the ROC data exhibit a linear trend in normal-probability space; practically all data points lie between the theoretical ROC functions associated with $d^{\prime}$ values of 0.7 and 1.0 (i.e., a range of 0.3 $d^{\prime}$ units). These trends suggest that the subjects made decisions using a cutoff or decision criterion, the value of which was varied in accordance with the payoffs. Analysis of the data from individual sessions showed that the criterion-based strategy appeared at very early stages of the training. By the 8th session, this strategy dictated about $90 \%$ of the responses. The major discrepancies with the signal-detection model resulted from use of suboptimal and unstable empirical criteria, particularly in pure statistical decision. In most cases, the subjects were too conservative with the payoff matrices that required extreme criterion values. ${ }^{6}$ In addition, they violated the criterionbased decision rule in about $10 \%$ of the trials on average.

Response-latency functions. Figure 3 (panels labeled A) shows latency functions obtained in the statisticaldecision and labeling tasks averaged across subjects. The average latency of the correct responses, $(\mathrm{a} / \mathrm{A}$ and $\mathrm{b} / \mathrm{B})$, is shown as a function of the value of the two-digit number. Response latencies ranged from 700 to $950 \mathrm{msec}$ and were remarkably similar across decision tasks; small differences between the functions were due partly to differences in the criterion values used in the tasks.

Two major latency effects can be observed. First, for numbers in the vicinity of the criterion values, response latency increases as the distance between the number stimulus and the criterion decreases. This effect ranges from 200 to $250 \mathrm{msec}$ on average. Table 2 reports Spearman rank correlations between latency and distance from cri- terion for each of three subjects. Negative correlations were obtained in all conditions, and 16 out of 18 coefficients were significant $(p<.05)$.

Second, for lax and strict criteria, latencies were slightly longer $(50-80 \mathrm{msec})$ for the less probable response-that is, for " $a$ " responses with lax criterion and for " $b$ " responses with strict criterion. In other words, for nonneutral criteria, the latency function is not exactly symmetric around the criterion: as distance from criterion increases, latencies for high-probability responses appear to decay faster than for low-probability responses. Wilcoxon signed-rank tests applied to the differences in latency between high- and low-probability responses to matched stimulus pairs (i.e., stimuli at the same distance above and below the lax or the strict criterion) are summarized in Table 2. In pure statistical decision, four out of six differences were significant $(p<.05)$; however, in labeling, none of the differences was significant. It is worth noting that, with lax and strict criteria, there is only a small number of matched stimulus pairs, and the signedrank tests are based on no more than three to five values. The small number of values might have worked against finding significant differences.

The labeling latency functions are similar to data obtained by Link (1990) in paired-comparison tasks with numbers sampled from the range between 11 and 99 . In Link's experiments, a standard and a comparison number were flashed in rapid succession, and subjects had to decide whether the comparison number was larger or smaller than the standard. Response times were related inversely to the numeric difference between the standard and comparison. In addition, for standards smaller than 55, the average latency of "smaller" responses was longer than that of "larger" responses; the reverse occurred with standards greater than 55. In other words, for midrange standards, response latencies were symmetric around the standard, and they were asymmetric for standards close to the endpoints of the numeric range. These trends were obtained with both rectangular and triangular number distributions.

\section{EXPERIMENT 2}

Although reasonably unambiguous, the results of Experiment 1 were not definitive because the subjects occasionally failed to use criterion values close to those of the optimal criteria. As a result, the peaks in the latency functions for lax and strict criteria tended to be close to the center of the decision continuum (or to the neutral criterion). In a few cases, the locations of the latency peaks for lax and strict criteria were not very different from the neutral criterion peak. Therefore, Experiment 2 was a replication of Experiment 1, except that the subjects had formal training in statistics. It was suspected that subjects who knew more about optimal decision strategies would employ criterion values more extreme than those of the high school students of Experiment 1.

All 4 subjects in Experiment 2 were unpaid volunteers who had completed two or more college-level courses in statistics and held a PhD degree. The apparatus and pro- 


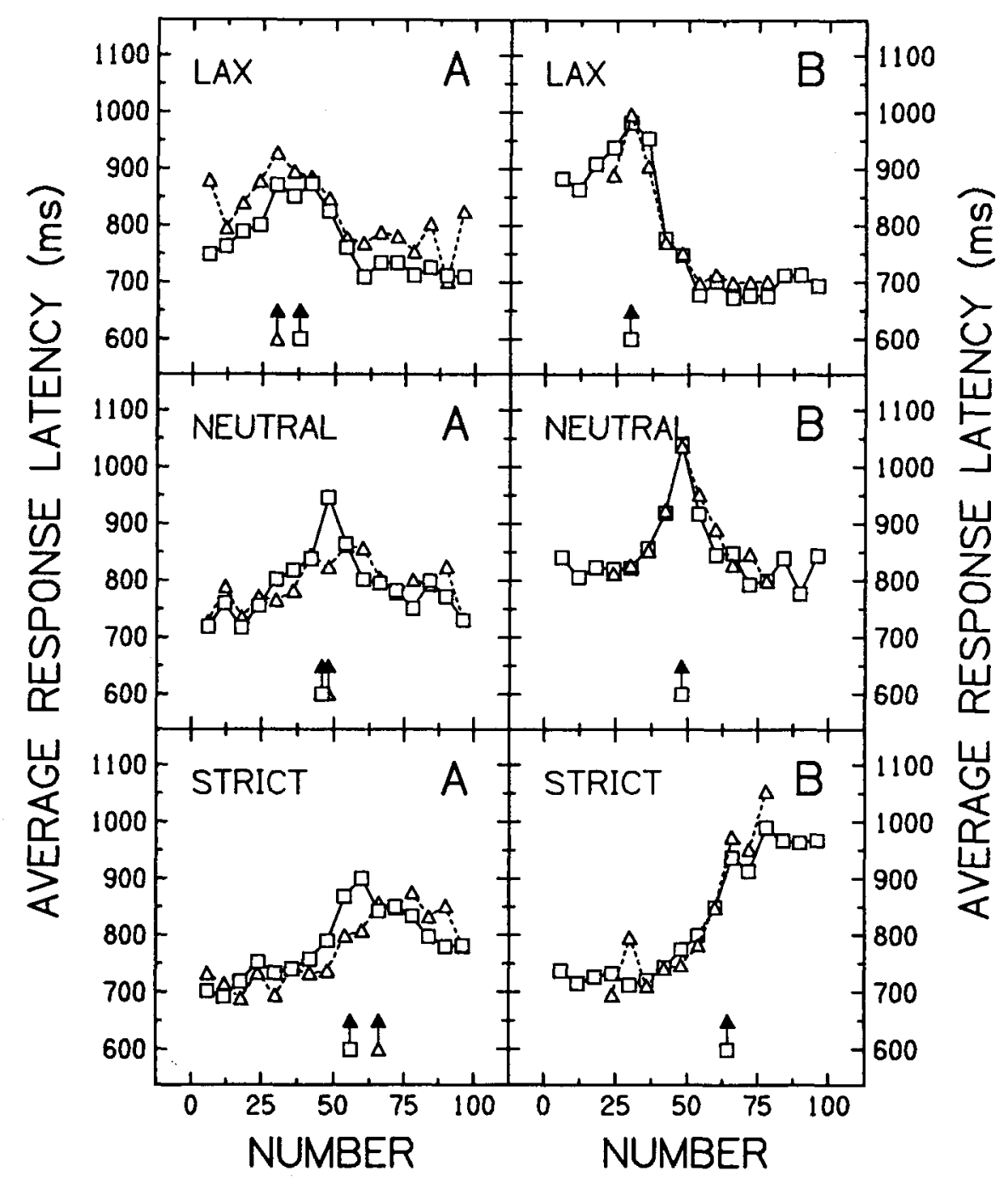

Figure 3. Average response latency plotted as a function of the value of the two-digit number. Data obtained with three payoff matrices are shown: lax (top panels), neutral (center panels), and strict (bottom panels). Panels labeled A show data for subjects without training in statistics (Experiment 1). Panels labeled B show data for subjects with training in statistics (Experiment 2). In panels labeled $A$, the different functions show data for pure statistical decision (open squares) and for labeling (open triangles). In panels labeled $B$, different functions show data for correct (open squares) and incorrect (open triangles) responses under pure statistical decision. For each decision task, arrows show the values of the empirical decision criteria averaged across subjects.

cedure were identical to those of Experiment 1, except that a longer response interval $(2,000 \mathrm{msec})$ was used. The subjects were tested for three sessions (2,700 trials) in the pure statistical decision task.

\section{Results}

Operating characteristics. The ROC data for each subject are shown in Figure 2 (panel labeled B). The data closely approach a straight line, and the observed $d^{\prime}$ values are nearly identical to the optimal value of 1.1 (see Table 1).
Response latency functions. The average response latencies for correct (open squares) and incorrect (open triangles) responses are shown in Figure 3 (panels labeled B). The trends are similar to those of Experiment 1, but the latency peaks for different decision criteria tend to be somewhat farther apart. The locations of the latency peaks for lax and strict criteria are clearly different from that of the neutral criterion. The effects of both criterion and response probabilities were larger in Experiment 2 than in Experiment 1. Table 3 summarizes the results of statistical analyses. Spearman rank correlations be- 
Table 2

Summary Statistics Experiment 1

\begin{tabular}{|c|c|c|c|c|c|}
\hline \multirow[b]{2}{*}{ Subject } & \multicolumn{3}{|c|}{ Spearman Rank Correlations } & \multicolumn{2}{|c|}{ Wilcoxon Signed-Rank Test } \\
\hline & Lax & Neutral & Strict & Lax & Strict \\
\hline \multicolumn{6}{|c|}{ Labeling } \\
\hline $\begin{array}{l}1 \\
2 \\
3\end{array}$ & $\begin{array}{l}-.196 \\
-.863^{*} \\
-.676^{*}\end{array}$ & $\begin{array}{l}-.634^{*} \\
-.551^{*} \\
-.621^{*}\end{array}$ & $\begin{array}{l}-.902^{*} \\
-.548^{*} \\
-.817^{*}\end{array}$ & $\begin{array}{r}-2.5 \\
-4.5 \\
0.5\end{array}$ & $\begin{array}{r}6.5 \\
-0.5 \\
7.5\end{array}$ \\
\hline \multicolumn{6}{|c|}{ Pure Statistical Decision } \\
\hline $\begin{array}{l}1 \\
2 \\
3\end{array}$ & $\begin{array}{l}-.760^{*} \\
-.810^{*} \\
-.564^{*}\end{array}$ & $\begin{array}{l}-.769 * \\
-.894^{*} \\
-.266\end{array}$ & $\begin{array}{l}-.823^{*} \\
-.797^{*} \\
-.550^{*}\end{array}$ & $\begin{array}{r}14.0^{*} \\
-6.5 \\
-12.0^{*}\end{array}$ & $\begin{array}{c}13.0^{*} \\
-4.5 \\
17.0^{*}\end{array}$ \\
\hline
\end{tabular}

Table 3

Summary Statistics for Pure Statistical Decision in Experiment 2

\begin{tabular}{cllllcc}
\hline & \multicolumn{2}{c}{ Spearman Rank Correlations } & & \multicolumn{2}{c}{ Wilcoxon Signed-Rank Test } \\
\cline { 2 - 3 } \cline { 6 - 7 } Subject & Lax & Neutral & Strict & & Lax & Strict \\
\hline 1 & $-.652^{*}$ & -.323 & $-.640^{*}$ & & -6.5 & -5.5 \\
2 & -.393 & $-.592^{*}$ & $-.532^{*}$ & & $-10.5^{*}$ & $-10.5^{*}$ \\
3 & $-.747^{*}$ & $-.574^{*}$ & $-.890^{*}$ & & $-10.5^{*}$ & $-10.5^{*}$ \\
4 & $-.623^{*}$ & -.450 & $-.618^{*}$ & & -7.5 & 5.0 \\
\hline${ }^{*} p<.05$. & & & & & & \\
\end{tabular}

tween latency and distance from criterion were negative in all cases, and 9 out of 12 coefficients were significant $(p<.05)$. The difference in latency between the highand low-probability responses was significant in four out of eight cases.

The shifts in criterion values and in response probabilities are closely related; however, each of these factors appears to have a somewhat different effect on the latencies: stimuli located at opposite sides of the criterion, but equidistant from it, have different latencies depending on whether the response has high or low probability.

The latencies of correct and incorrect responses show no systematic differences. This result provides an estimate of the reliability of the latency measures, since there is no physical difference between a particular number stimulus when it is defined (correctly or not) as an " $a$ ", or a "b" sample. It should be noted that close similarity in latencies of correct and incorrect responses is obtained only with a stimulus-by-stimulus analysis of performance. The latencies of incorrect responses are considerably longer than those for correct responses when overall averages are compared. As noted earlier, most incorrect responses occur with stimuli that are close to the criterion.

Interindividual differences. Figure 3 shows average latencies for the group of subjects tested in each experiment. The size of interindividual differences is illustrated in Figure 4, which shows latency functions for each subject of Experiment 2 (pure statistical decision task). Individual differences in response latency and in the location of the decision criteria are easily seen. Nevertheless, the trends observed in the average functions (Figure 3) are seen also in the individual data: all 4 subjects showed effects of criterion differences and of response probability.
In interpreting the data of Figures 3 and 4, a feature of the stimulus statistics must be considered: the number of trials used to compute average latencies is different for each bin of the decision continuum, and the number decreases sharply toward the extremes of the continuum. This feature is inherent to the statistics of sensory events as modeled by the theory of signal detectability, TSD (Green \& Swets, 1966), and is shared by all the experiments reported here. The decrease in the number of trials could be producing longer latencies toward the endpoints of the decision axis. It is well known that reaction time increases inversely with the a priori probability of the signal (e.g., Laming, 1968), although the effect can be attenuated or eliminated by practice (Mowbray $\&$ Rhoades, 1959). All the results reported in this study were obtained after prolonged training $(15,000-25,000$ trials), with the exception of Experiment 2 in which the subjects were tested for 2,700 trials. However, in some instances (for example, see Figure 4, neutral criterion), latencies tend to increase at the endpoints of the decision continuum. It is appropriate to consider possible effects of the unequal number of trials along the stimulus continuum.

First, it is clear that longer response latencies near the criterion cannot be attributed to stimulus probability: latencies varied with criterion changes, although the a priori stimulus probabilities were identical across payoff matrices. Second, whenever the subject adopts a nonneutral criterion, both the less likely response and the associated stimuli have low probability. The longer latency of the less frequent response may result partly from low stimulus probability, low response probability, or a combination of both. However, both endpoints of the decision continuum have low stimulus probability, but only one 


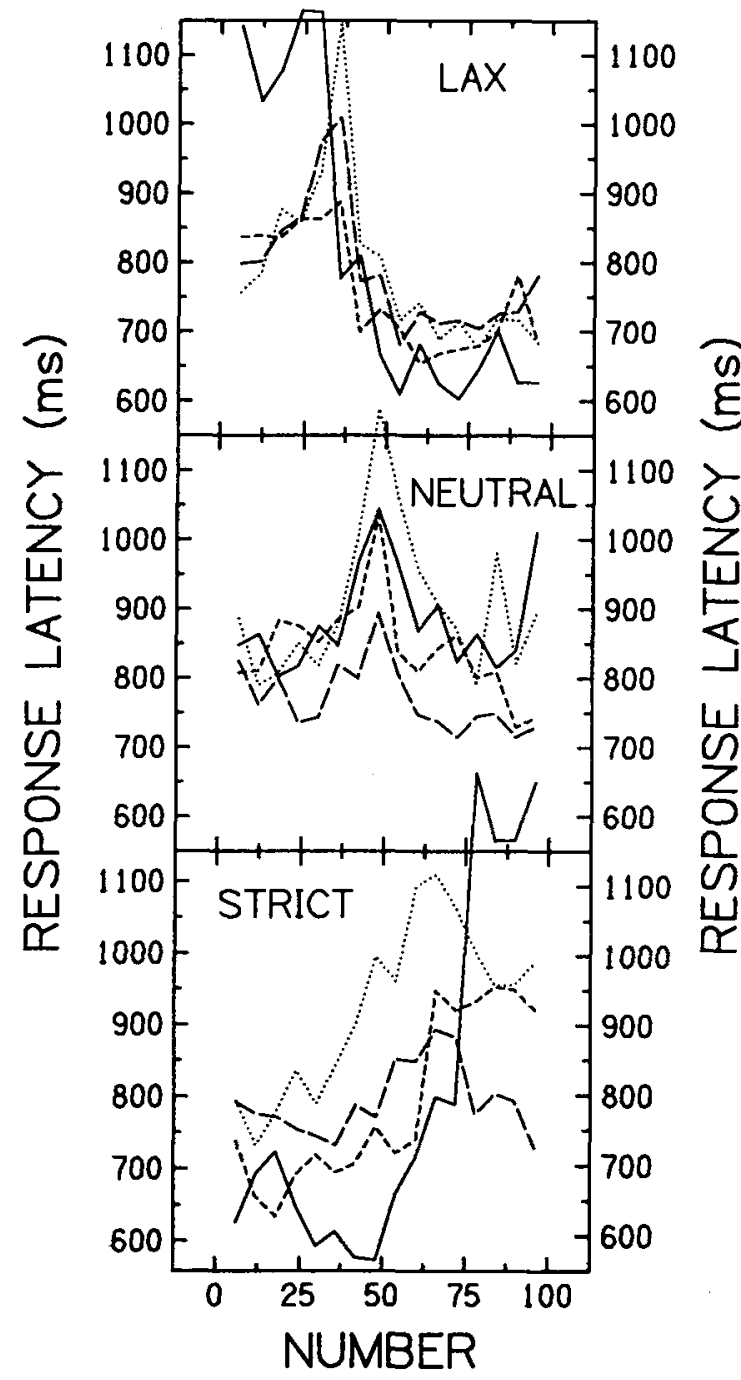

Figure 4. Response latencies for individual subjects in Experiment 2. Within each panel the functions relate response latency (ordinate) to values of number (abscissa), with individual subjects as parameter. Panels from top to bottom are for lax, neutral, and strict payoff matrices.

endpoint exhibits long latencies with nonneutral criteria. Therefore, response probability seems more influential than stimulus probability in producing long latencies. The unequal number of trials along the stimulus continuum seems to have only a second-order effect on the latencies. The preceding conclusions should not be interpreted to mean that stimulus and response probability uniquely predict response latencies. Differences in response latencies may be attributed also to one response requiring more information than the other (e.g., Ratcliff, 1985), but the role of this factor was not examined in the present experiments.

\section{EXPERIMENT 3}

The next two experiments extended the previous work to the case of auditory stimuli and to an additional variation of the subject's task. The stimuli were distributions of tonal frequencies. In Experiment 3, the frequency range of each distribution was greater than an octave, and the individual tones could all be discriminated without difficulty. In Experiment 4, the frequency range of each distribution was smaller than an octave, and differences among many of the tones were below the frequency difference limen.

\section{Method}

Subjects. The experiment was carried out in two replications with 3 subjects in each. In the first, the subjects were the same as those of Experiment 1. In the second, the subjects were male high school students (15 years old on the average), with normal hearing, and without formal background in statistics or experience in psychophysical experiments. All subjects were paid for their participation.

Apparatus. A standard auditory signal generation system was used, with an oscillator, noise generator, electronic switch, and a bandpass filter. The stimulus presentation, recording of responses, and all other experimental events were controlled by a PDP-8/S computer. The subjects worked individually in sound-treated booths. The stimuli were presented monaurally to the right ear (Telephonics TDH -49 headphones) at $75 \mathrm{~dB}$ SPL.

Procedure. The distributions of tonal frequencies had the same statistical properties as those of the numbers used in Experiments 1 and 2. The tones covered a range from 500 to $1550 \mathrm{~Hz}$. A series of tonal frequencies was generated by addition of a constant proportional increment $(\Delta f / f=0.012)$ to each successive term, beginning with $500 \mathrm{~Hz}$. This resulted in 96 equi-log steps within the 500 $1550-\mathrm{Hz}$ frequency range, with a frequency difference equal to or greater than $6 \mathrm{~Hz}$ between any two adjacent steps. Each frequency step was treated in the same way as the numbers of Experiments 1 and 2 , and the same procedure for constructing stimulus distributions was followed. The difference between the midpoints of two consecutive (six-element) categories or bins was equal to or greater than $32 \mathrm{~Hz}$. The distance between the means of the $A$ and $B$ distributions was approximately $191 \mathrm{~Hz}\left(X_{\mathrm{A}}=791 \mathrm{~Hz} ; X_{\mathrm{B}}=982 \mathrm{~Hz}\right)$. The A distribution ranged from 500 to $1237 \mathrm{~Hz}$, and the B distribution ranged from 619 to $1550 \mathrm{~Hz}$.

In order to investigate each of the three decision tasks described in the General Method section, the trial structure was modified slightly. Two 200 -msec auditory events were presented on each trial, separated by $250 \mathrm{msec}$. The first one (called the standard) could be either noise (100-3000 Hz bandwidth; $66 \mathrm{~dB}$ spectrum level) or a 75-dB-SPL pure tone with one of three alternative frequencies: 710,880 , or $1091 \mathrm{~Hz}$. These frequencies corresponded to the values of the optimal lax, neutral, and strict criteria, respectively, for each of the payoff matrices used in Experiments 1 and 2. During a given 100-trial block, either noise or one of the three standard (criterion) tones was presented. The second event (termed comparison) was a pure tone sampled from one of the two distributions. The sampling was random and without replacement, following the same rules of the experiments with numbers. The subjects' task was to decide from which distribution the comparison tone was sampled. The comparison tone was followed by a 1,750-msec response interval. The onsets of the comparison tones of consecutive trials were separated by a $3,050-\mathrm{msec}$ interval. The response latencies were measured from the onset of the comparison tone.

The subjects were tested in all three decision tasks. For instructed statistical decision and labeling, the presentation of each comparison tone was preceded by the corresponding standard tone. For pure statistical decision, the standard tone was replaced by noise. The goals, instructions, and other aspects of the different tasks were defined as in the previous experiments. Again, prior to beginning the experiments, the characteristics of the stimulus sets and the nature of the tasks were carefully explained to the subjects.

Each experimental session consisted of six to eight 100-trial blocks, separated by rest periods. The first replication included 22 
experimental sessions. During the first 19 sessions, the three decision tasks varied from session to session in a counterbalanced order. During the last 3 sessions, the tasks varied between blocks of trials within a session as a further control for practice effects. The second replication included 32 sessions. The first 8 were devoted to practice, with one decision task per session. In the remaining 24 sessions, the three decision tasks varied between blocks of each session.

\section{Results}

Operating characteristics. One subject dropped out of the experiment by the 8th session, and the results were discarded. Figure 2 (panels labeled C) shows the ROC data for each subject. The data points were obtained from the final 14 sessions of the first replication and from the final 23 sessions of the second one. The subjects required 8-10 sessions to reach performance levels similar to those of the experiments with numbers. It can be seen again that, with all three tasks, each subject's performance was very close to the optimum. In these highly trained listeners, replacing the standard tone with noise did not degrade performance by a large amount. In addition, a comparison of Panels A and B with Panels C of Figure 2 reveals that performance in the present experiment was only slightly lower than that in the experiments with numbers.

Response latency functions. The latency functions averaged across subjects are shown in Figure 5 (panels

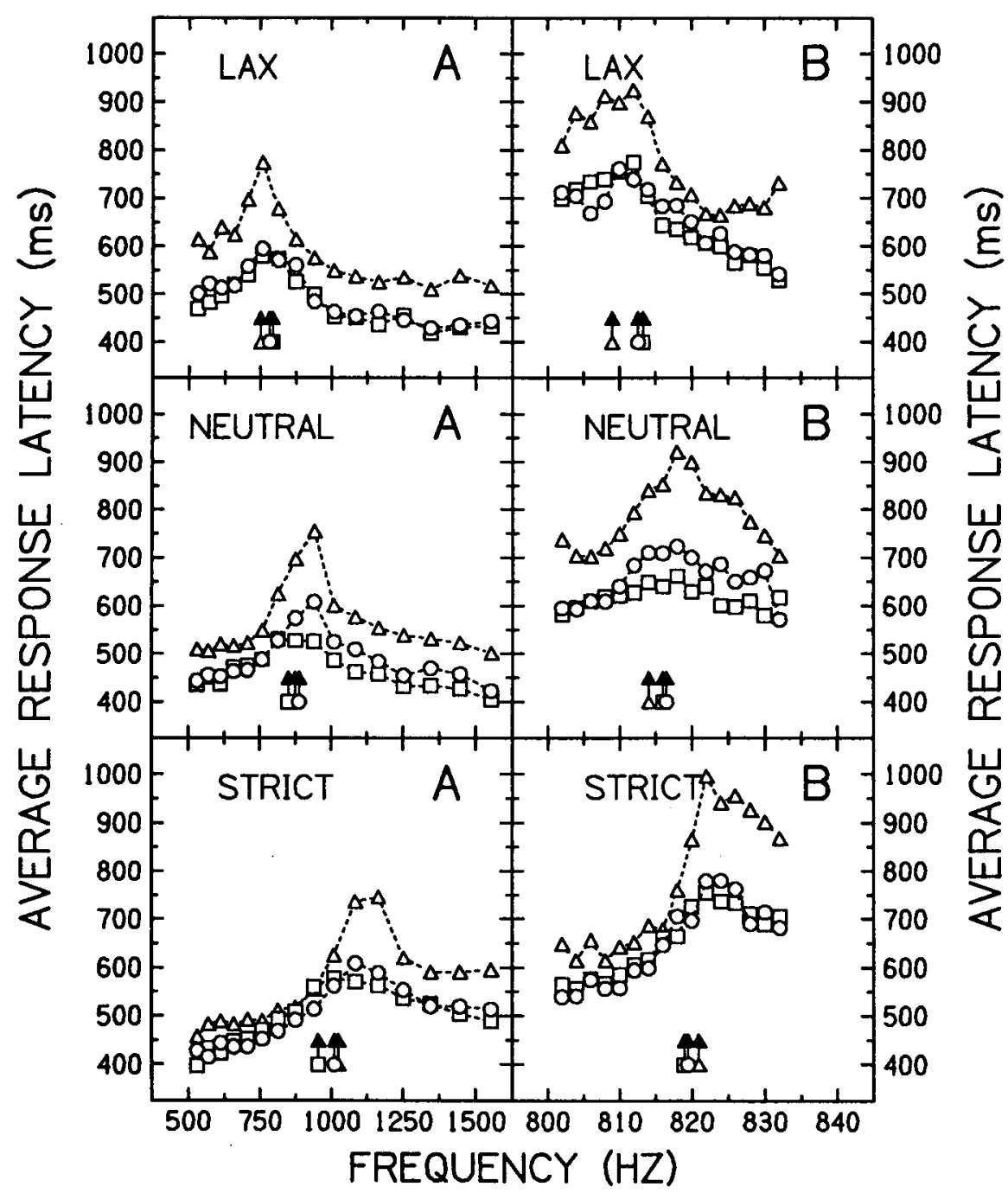

Figure 5. Average response latency plotted as a function of tonal frequency. Data obtained with high-discriminability tones (panels labeled $A$ ) and with low-discriminability tones (panels labeled B) are shown. Panels from top to bottom are for lax, neutral, and strict payoff matrices. Within each panel, the parameter is the decision task: pure statistical decision (open squares), instructed statistical decision (open circles), and labeling (open triangles). Arrows show the locations of the decision criteria along the frequency decision axis. Note that the ordinate scale of this figure is different from that of Figures 3 and 4. 
Table 4

Summary Statistics Experiment 3

\begin{tabular}{|c|c|c|c|c|c|}
\hline \multirow[b]{2}{*}{ Subject } & \multicolumn{3}{|c|}{ Spearman Rank Correlations } & \multicolumn{2}{|c|}{ Wilcoxon Signed-Rank Test } \\
\hline & Lax & Neutral & Strict & Lax & Strict \\
\hline \multicolumn{6}{|c|}{ Labeling } \\
\hline 1 & $-.910^{*}$ & -.474 & $-.731^{*}$ & 2.5 & 7.5 \\
\hline 2 & $-.800 *$ & $-.900 *$ & $-.809 *$ & 1.5 & 7.5 \\
\hline 3 & $-.847^{*}$ & $-.812 *$ & $-.774^{*}$ & 2.0 & 11.0 \\
\hline 4 & $-.827^{*}$ & -.419 & -.340 & -6.5 & $10.5^{*}$ \\
\hline 5 & $-.641 *$ & $-.579 *$ & $-.503 *$ & -9.5 & 7.5 \\
\hline \multicolumn{6}{|c|}{ Pure Statistical Decision } \\
\hline 1 & $-.915^{*}$ & $-.959 *$ & $-.979 *$ & $-10.5^{*}$ & -7.5 \\
\hline 2 & $-.935^{*}$ & -.227 & $-.624^{*}$ & 2.5 & 6.5 \\
\hline 3 & $-.929 *$ & $-.894 *$ & $-.517^{*}$ & $-16.0^{*}$ & $18.0^{*}$ \\
\hline 4 & -.496 & -.450 & -.242 & $-14.0^{*}$ & $14.0^{*}$ \\
\hline 5 & $-.764 *$ & $-.782 *$ & -.097 & -7.5 & $14.0 *$ \\
\hline \multicolumn{6}{|c|}{ Instructed Statistical Decision } \\
\hline 1 & $-.759 *$ & $-.923 *$ & $-.803^{*}$ & $-16.0^{*}$ & 6.5 \\
\hline 2 & $-.809 *$ & $-.564 *$ & $-.838^{*}$ & $-3.5^{*}$ & 7.5 \\
\hline 3 & $-.912 *$ & $-.744 *$ & $-.588 *$ & -3.0 & $14.0 *$ \\
\hline 4 & $-.696 *$ & -.489 & -.194 & $-10.5^{*}$ & $10.5^{*}$ \\
\hline 5 & $-.629^{*}$ & $-.682 *$ & -.372 & $-10.5^{*}$ & $10.5^{*}$ \\
\hline
\end{tabular}

labeled A). For ease of comparison, data from the three strategies collected after similar amounts of practice are included in each panel. The average response latencies ranged from 400 to $800 \mathrm{msec}$-that is, latencies were shorter than those obtained with numbers. In other respects, the functions exhibit trends similar to those described in the previous experiments: latencies show a maximum at the decision criterion and are slightly longer for the response with lower probability. In this experiment, the response probability effect was much smaller than that in Experiment 2. Results of statistical tests appear in Table 4. All 45 Spearman rank correlations between latency and distance from criterion were negative, and 34 coefficients were significant $(p<.05)$. Out of 10 signed-rank tests computed for each decision task, 1, 6, and 7 reached significance, for labeling, pure, and instructed statistical decision, respectively.

As in the previous experiments, the shape of the latency functions was similar across decision tasks. The only clear difference was that labeling latencies were longer than those for statistical decisions (sometimes by as much as $150 \mathrm{msec}$ ). This effect was not due to differences in amount of task training.

\section{EXPERIMENT 4}

This experiment examined effects of criterion on response latency using stimulus conditions comparable to those of standard signal-detection experiments.

\section{Method}

Subjects. Two male and 2 female high school students (17.5 years old on average), with normal hearing sensitivity, participated in the study. None of them had experience in psychophysical ex- periments nor any formal background in statistics. They were paid for their participation.

Apparatus and Procedure. The apparatus was the same as that in Experiment 3. The distributions of tones were generated as before, but this time they covered a range from 800 to $832 \mathrm{~Hz}$. A series was generated by adding a constant proportional increment ( $\Delta f / f=0.00041$ ) to each successive term, beginning with $800 \mathrm{~Hz}$. This resulted in 96 equi-log steps within the frequency range used, with a frequency difference $\geq 0.33 \mathrm{~Hz}$ between two successive steps. The frequency difference between the midpoints of two consecutive (six-element) bins or categories was $\geq 2.0 \mathrm{~Hz}$. The distance between the means of the distributions was approximately $7 \mathrm{~Hz}$ $\left(X_{\mathrm{A}}=812 \mathrm{~Hz}, 800-825 \mathrm{~Hz}\right.$ range; $X_{\mathrm{B}}=819 \mathrm{~Hz}, 806-832 \mathrm{~Hz}$ range), which corresponds to $\Delta f / f=0.0071$. The stimulus differences in the present experiment approximated those of a standard frequency discrimination task. As in the previous experiments, the ideal $d^{\prime}$ was 1.1 , but subjects' performance could be lower than the ideal value. The efficiency index (i.e., the ratio between the obtained and ideal $d^{\prime}$ values) was expected to be lower in this experiment than in the previous one. The trial structure and decision tasks were the same as in the previous experiment.

The subjects were tested for a total of 29 sessions. The first 13 were practice sessions, again to allow the subjects to reach accuracy levels comparable to those of the previous experiments. In the remaining 16 sessions, the pure and instructed statistical decision tasks were used in alternate blocks within a session. To reduce the complexity of the experiment, the labeling task was not employed after the 13th session.

\section{Results}

Operating characteristics. In the analysis of the results, the data from one subject were discarded because her decision criteria were very unstable, yielding low accuracy levels. The ROC data for the other 3 subjects are shown in Figure 2 (panels labeled D). In general, in all three tasks the ROC data approach straight lines in normalprobability space, and the values of $d^{\prime}$ are only slightly lower than 1.0-that is, accuracy was only slightly lower 
Table 5

Summary Statistics Experiment 4

\begin{tabular}{|c|c|c|c|c|c|}
\hline \multirow[b]{2}{*}{ Subject } & \multicolumn{3}{|c|}{ Spearman Rank Correlations } & \multicolumn{2}{|c|}{ Wilcoxon Signed-Rank Test } \\
\hline & Lax & Neutral & Strict & Lax & Strict \\
\hline \multicolumn{6}{|c|}{ Labeling } \\
\hline 1 & $-.859 *$ & $-.862^{*}$ & $-.729 *$ & $-10.5^{*}$ & $10.5^{*}$ \\
\hline 2 & $-.709 *$ & $-.734 *$ & $-.823^{*}$ & -5.0 & $10.5^{*}$ \\
\hline 3 & $-.827^{*}$ & $-.540 *$ & $-.747^{*}$ & -5.0 & 5.0 \\
\hline \multicolumn{6}{|c|}{ Pure Statistical Decision } \\
\hline 1 & -.424 & $-.514^{*}$ & $-.543^{*}$ & $-14.0^{*}$ & $14.0^{*}$ \\
\hline 2 & $-.651^{*}$ & $-.812^{*}$ & $-.582 *$ & $-10.5^{*}$ & $14.0^{*}$ \\
\hline 3 & $-.777^{*}$ & $-.981^{*}$ & $-.779^{*}$ & $-14.0^{*}$ & $10.5^{*}$ \\
\hline \multicolumn{6}{|c|}{ Instructed Statistical Decision } \\
\hline 1 & $-.509^{*}$ & $-.541^{*}$ & $-.856^{*}$ & $-10.5^{*}$ & $10.5^{*}$ \\
\hline 2 & $-.771^{*}$ & $-.627^{*}$ & $-.562^{*}$ & $-10.5^{*}$ & $13.0^{*}$ \\
\hline 3 & $-.924 *$ & $-.830^{*}$ & $-.716^{*}$ & 1.0 & $10.5^{*}$ \\
\hline
\end{tabular}

than in the previous experiment. No other important differences were observed between the ROC data of Experiment 4 and those of Experiment 3. On average, the violation rates in Experiment 4 were 13\% for labeling, 16\% for instructed, and $21 \%$ for pure statistical decision. These values are only slightly larger than those observed in Experiment 3 with tones $(6 \%, 10 \%$, and $16 \%$, respectively).

Response latency functions. Figure 5 (panels labeled B) shows latency functions for the three tasks averaged across subjects. On average, latencies ranged from 500 to $1,000 \mathrm{msec}$, and therefore were considerably longer than the latencies of Experiment 3. Very likely, this was a consequence of having increased the task difficulty, while attempting to maintain high accuracy. The effects of decision criterion and of response probability are very similar to those of the previous experiments. However, in Experiment 4, the response probability effect was greater than in Experiment 3. Again, the only clear difference between decision tasks was that the latencies were longer for labeling than for statistical decisions. Although the labeling data were collected after about 5,400 practice trials, the possibility of a training effect cannot be ruled out.

Table 5 summarizes the results of statistical tests. All 36 Spearman rank correlations were negative, and only 1 failed to reach significance. Out of 6 signed-rank tests computed for each decision task, 3, 6, and 5 reached significance for labeling, pure, and instructed decision, respectively. In Experiments 1, 3, and 4, the latency effects of response probability were more robust in statistical decisions than in labeling.

Experiments 3 and 4 showed that the results obtained with numbers are obtained also with auditory stimuli having either high or low discriminability. Reduced discriminability of the tonal frequencies caused an overall increase in the response latencies, but the shape of the function remained much the same with the three decision tasks. These experiments also showed that, although reduced in size, the latency effects are not abolished by prolonged training.

\section{SUMMARY OF RESULTS}

The present study had three main results:

(1) In binary decision tasks, response latency was inversely related both to the distance between the stimulus and criterion and to the probability of the response. The criterion effect was both large and highly consistent: out of a total of 102 individual latency functions, 85 yielded statistically significant negative correlations. The response-probability effect was clearly smaller and less consistent than the criterion effect: for labeling, the effect was significant in 4 out of 22 cases; for statistical decisions, 32 out of 44 cases were significant. The latency effects of criterion and response probability were interrelated, but they appear to be different: with nonneutral criteria, the latency function was not exactly symmetric around the criterion. Because response probabilities were determined by the decision criterion, it is valid to conclude that the criterion was the primary determinant of response latencies of binary decisions.

(2) The trends described above were observed with different stimulus modalities (tones and numbers), different discriminability levels within the auditory modality, and different decision tasks. In particular, the relations among stimulus, decision criterion, and latency were nearly the same whether or not the decision task required the observer to refer to stimulus probabilities or to payoff matrices to make binary decisions.

(3) Although the general trend of the results was very similar across experimental conditions, the size of the effects varied with the subject, stimulus modality, discriminability level (for tone frequencies), and amount of practice. The reported effects were observed even after considerable training in the tasks.

\section{DISCUSSION}

\section{Stimulus Statistics}

It is appropriate to discuss briefly the relation between the present decision tasks and the typical TSD situation 
in which signals near the discrimination or detection threshold are used. The central issue deals with the characteristics of the stimulus distributions used in this study. Although consisting of discrete stimulus values, these distributions closely approximated a Gaussian function. In fact, the area proportions above and below a given $z$-score value are almost identical to those of a continuous normal distribution. However, the variances of the distributions used in the present study were considerably larger than those typically found in standard detection or discrimination experiments. The distance between the distribution means was correspondingly large, so that optimal discriminability was about $d^{\prime}=1.0$, in all cases.

Use of these kinds of stimulus distributions in the analysis of decision processes has the following advantages: (1) they enhance the effects of the physical differences between the individual stimuli of the distributions, (2) they presumably reduce the detection/discrimination demands imposed upon the sensory system, and (3) they facilitate a trial-by-trial analysis of performance and a more detailed and less inferential description of the observer's strategies. Previous studies have shown that the stimuli and tasks used in this study provide adequate models of standard signal-detection experiments (e.g., Kubovy et al., 1971; Lee \& Janke, 1964; Ratcliff, 1987).

\section{Factors Influencing Response Latencies of Binary Decisions}

Response latencies varied inversely with the distance between the stimulus and the current criterion and, to a lesser extent, with the probability of the response elicited by the stimulus. These results are in general agreement with previous indirect evidence obtained in detection studies (e.g., Carterette et al., 1965; Emmerich et al., 1972; Gescheider et al., 1968; Gescheider et al., 1969; Norman \& Wickelgren, 1969), and with theoretical predictions (e.g., Bindra, Williams, \& Wise, 1965; Laming, 1968; Link, 1990; McGill, 1963; Nickerson, 1969; Ratcliff, 1985; Sekuler, 1965; Smith, 1968; Stone, 1960; Thomas, 1971; Thomas \& Myers, 1972). Compared with previous work, the main contribution of the present study is in providing a detailed description of the relation between response latencies, values of sensory inputs along the decision axis, decision criterion, and response probabilities. The detailed relation between these variables has been hypothesized or inferred in previous studies, but not measured experimentally. Several of these hypotheses (e.g., Navon, 1975, p. 62; Pike, 1973, p. 56; Ratcliff, 1987; Ratcliff \& Hacker, 1981; Vickers, 1979) seem partly incorrect or incomplete in view of the present results. For example, previous studies have assumed that changes in latency caused by criterion shifts are identical to those caused by shifts in response probability (e.g., Emmerich et al., 1972; Gescheider et al., 1968; Gescheider et al., 1969; see also Ratcliff, 1987). The present experiments suggest that the effects of these two variables on response latencies may not be necessarily equivalent. Stimuli located at opposite sides of the criterion, but equidistant from it, have different latencies depending on whether the response has high or low probability.

The pattern of response latencies obtained with neutral criterion (i.e., no response bias) is consistent with models predicting latency functions that are symmetric around the decision criterion, such as the runs (Audley, 1960) and random-walk (Laming, 1968; Stone, 1960) models. The asymmetric latency functions predicted by other models are in conflict with our data. The latter include the accumulator (Vickers, Caudrey, \& Willson, 1971) and recruitment (La Berge, 1962) models.

\section{Processes Underlying Sensory Decisions}

In all four experiments, the ROC data and response latencies are consistent with the hypothesis that sensory decisions were made on the basis of whether or not stimuli exceeded a criterion value. A related issue concerns the sensory-decision variable along which the sensory input and the criterion are compared. A comparison of results from pure and instructed statistical decision with those of labeling provides insights into this question. With labeling, the subjects did not need to know the stimulus statistics, likelihood ratios, or payoff matrices. The results showed that performance, in terms of $d^{\prime}$ units, was very similar across decision tasks. Minor differences were obtained in the $d^{\prime}$ values and in the location and stability of the criteria.

It is reasonable to assume that with labeling, the decision variable is the simple perceptual distance or similarity between the standard (criterion) and the comparison stimulus (sensory input). In addition, this type of processing appears to be the simplest-that is, the one that would require minimum recoding of the input. Therefore, labeling latencies would be expected to be shorter than those of statistical decisions. The results showed labeling latencies to be either approximately equal to (Experiment 1) or longer than (Experiment 3) those of statistical decisions. The shape of the functions was similar across tasks. These results would seem to suggest that in statistical decisions, the variable along which sensory inputs and criteria are compared may also be simple perceptual distance.

A model of the decision process that relies on perceptual distances is more akin to the threshold energy detection model (Green \& Swets, 1966; McGill, 1967) than to the more conventional TSD model. Conventional TSD states that the observer's decision variable is a monotonic transform of the likelihood ratio associated with each sensory input (Green \& Swets, 1966, p. 31). The observer would convert the stimulus into a cognitive equivalent of the likelihood ratio (Luce, 1963, p. 109; McGill, 1967). Apparently, as suggested by McGill (1967), the mechanisms underlying sensory decisions are much more primitive than those hypothesized by TSD (e.g., Edwards, 1965; Laming, 1968). The comparison between sensory input and criterion seems to be made using fairly basic properties of the stimuli rather than using highly recoded characteristics. Consistent with this interpretation is the fact that Link (1990) obtained results very similar to those 
of Experiments 1 and 2, although he employed tasks and number distributions somewhat different from those of the present study. Link's number-comparison theory also relies on perceptual distances between standard and comparison stimuli.

It is likely that information about stimulus statistics and decision outcomes is used to determine the criterion value that maximizes the decision goals; however, once this value is determined, it may well be that only perceived distance is used to decide whether or not an input exceeds the criterion. In other words, observers order the stimuli along the perceptual dimension relevant to the task (e.g., pitch) and respond on the basis of the difference between stimuli and criterion along this dimension. Further analyses have shown that the latency function tends to linearize when plotted against the logarithm of the difference between stimuli and criteria. A number of sensory dimensions (e.g., the mel scale for pitch) exhibit a linear relation with the logarithm of stimulus magnitude.

In conclusion, the present results suggest that the process of making a sensory decision may involve two basic operations, which are to a good degree independent: (1) determination of the decision criterion value and (2) determination of the relation between the current stimulus and the criterion. A similar distinction has been proposed by Ratcliff (1987), but considerably more research is needed to make definite conclusions on this matter.

\section{REFERENCES}

Audley, R. J. (1960). A stochastic model for individual choice behaviour. Psychological Review, 67, 1-15.

BINDRA, D., WILLIAMS, J. A., \& WISE, J. S. (1965). Judgments of sameness and differences: Experiments on decision time. Science, 150, 1625-1627.

Carterette, E. C., Freidman, M. P., \& Cosmides, R. (1965). Reaction-time distributions in the detections of weak signals in noise. Journal of the Acoustical Society of America, 37, 531-542.

Dorfman, D. D., \& ALF, E. (1968). Maximum likelihood estimation of parameters of signal detection theory: A direct solution. Psychometrika, 33, 117-124.

EDWARDS, W. (1965). Optimal strategies for seeking information: Models for statistics, choice reaction times and human information processing. Journal of Mathematical Psychology, 2, 312-329.

Emmerich, D. S., Gray, J. L., Watson, C. S., \& TaNis, D. C. (1972). Response latency, confidence, and ROCs in auditory signal detection. Perception \& Psychophysics, 11, 65-72.

Gescheider, G. A., Wright, J. H., \& Evans, M. B. (1968). Reaction time in the detection of vibrotactile signals. Journal of Experimental Psychology, 77, 501-504.

Gescheider, G. A., Wright, J. H., Weber, B. J., Kirchner, B. H., \& Milligan, E. A. (1969). Reaction times as a function of the intensity and probability of occurrence of vibrotactile signals. Perception \& Psychophysics, 5, 18-20.

GreEN, D. M., SWETS, J. A. (1966). Signal detection theory and psychophysics. New York: Robert E. Krieger.

Kubovy, M., RaPOPORT, A., \& TVERSKY, A. (1971). Deterministic versus probabilistic strategies in detection. Perception \& Psychophysics, 9, 427-429.

LA Berge, D. (1962). A recruitment theory of simple behavior. Psychometrika, 27, 375-396.

LAMING, D. R. J. (1968). Information theory and choice-reaction time. New York: Wiley.

LEE, W. \& J ANKE, M. (1964). Categorizing externally distributed stim- ulus samples from three continua. Journal of Experimental Psychology, 68, 376-382.

LiNK, S. (1990). Modelling imageless thought: The relative judgment theory of numerical comparisons. Journal of Mathematical Psychology, 34, 2-41.

LuCE, R. D. (1963). Detection and recognition. In R. D. Luce, R. R. Bush, \& E. G. Galanter (Eds.), Handbook of mathematical psychology (Vol. 1, pp. 103-190). New York: Wiley.

MCGILL, W. J. (1963). Stochastic latency mechanisms. In R. D. Luce, R. R. Bush, \& E. G. Galanter (Eds.), Handbook of mathematical psychology (Vol. 1, pp. 309-360). New York: Wiley.

McGilL, W. J. (1967). Neural counting mechanisms and energy detection in audition. Journal of Mathematical Psychology, 4, 351-376.

Mowbray, B. H., \& RHoades, M. V. (1959). On the reduction of choice reaction times with practice. Quarterly Journal of Experimental Psychology, 11, 16-23.

Navon, D. (1975). A simple method for latency analysis in signal detection tasks. Perception \& Psychophysics, 18, 61-64.

Nickerson, R. S. (1969). Same-different response times: A model and a preliminary test. In W. G. Koster (Ed.), Attention and performance II (pp. 257-275). London: North-Holland.

Norman, D. A., \& Wickelgren, W. A. (1969). Strength theory of decision rules and latency in short-term memory. Journal of Mathematical Psychology, 6, 192-208.

Pike, R. (1973). Response latency models for signal detection. Psychological Review, 80, 53-68.

RatcLIFF, R. (1985). Theoretical interpretations of the speed and accuracy of positive and negative responses. Psychological Review, 92 , 212-225

RATCLIFF, R. (1987). More on the speed and accuracy of positive and negative responses. Psychological Review, 94, 277-280.

RATCLIFF, R., \& HACKER, M. J. (1981). Speed and accuracy of same and different responses in perceptual matching. Perception \& Psychophysics, 30, 303-307.

SEKUlER, R. W. (1965). Signal detection, choice response times, and visual backward masking. Canadian Journal of Psychology, 19, 118-132.

SMITH, P. T. (1968). Cost, discriminability, and response bias. British Journal of Mathematical Statistical Psychology, 21, 35-60.

Stone, M. (1960). Models for choice-reaction time. Psychometrika, 25, 251-260.

Thomas, E. A. C. (1971). Sufficient conditions for monotone hazard rate: An application to latency-probability curves. Journal of Mathematical Psychology, 8, 303-332.

THOMAS, E. A. C., MYERS, I. C. (1972). Implications of latency data for threshold and non-threshold models of signal detection. Journal of Mathematical Psychology, 9, 253-285.

VICKERS, D. (1979). Decision processes in visual perception. New York: Academic Press.

Vickers, D., Caudrey, D., \& Willson, R. (1971). Discriminating between the frequency of occurrence of two alternating events. Acta Psychologica, 35, 151-172.

WATSON, C. S. (1962). Signal detection and certain physical characteristics of stimulus during the observation interval. Unpublished doctoral dissertation, Indiana University, Bloomington.

\section{NOTES}

1. The expressions response time, reaction time, response latency, and others are used in the literatuite with more than one meaning. We will use the expression response time as a generic term. Reaction time will designate situations in which the subject is under time pressure. Response latency will refer to situations in which the subject has no response-time restrictions and is not informed that response latency is being monitored.

2. In these experiments, the trial-by-trial feedback was needed to allow the subjects to learn the statistics of the stimulus distributions; however, for nonneutral criteria this feedback conflicted with the goal of maximizing either the expected gain or the percent correct labeling. For example, to achieve maximum gain with a strict criterion the subjects 
had to decrease their overall percent correct. With nonneutral criteria, the subjects were told to maximize the expected gain or the percent correct labeling, even if they felt that they were making more errors relative to the trial-by-trial feedback.

3. The adequacy of the trial structure was indicated by the following: (1) the average response time was rarely longer than $1,200 \mathrm{msec}$, (2) responding after the end of the response interval was considered as a miss response-the miss rate was less than $0.5 \%$-and (3) a longer response interval was used in Experiment 2, and it was found that the subjects did not use the extra time.

4. Although obviously simple, the labeling task was not trivial due to the following: (1) the number stimuli were chosen randomly and displayed for $265 \mathrm{msec}$, (2) the subjects did not know in advance which number would be presented on a given trial, and they had to compare the number stimuli with their memory representation of the criterion number, (3) the labeling decision was made in a brief period of time, and (4) the subjects had to select the appropriate response button. With pure-tone stimuli, the labeling task was more challenging than with numbers. In none of the experiments did labeling reach $100 \%$ correct. Given the above, it seems unlikely that the subjects may have performed a digit-by-digit comparison with the number stimuli (a review of mentalcomparison experiments with number stimuli appears in Link, 1990).

5. Although the values of the ROC area and violation rates are correlated, they are not identical. For example, by trial-to-trial criterion fluctuation, subjects can on occasion correctly respond " $b$ " to a B stimulus that is below the average criterion used within a session. This hit response violates the decision rule dictated by the average criterion. In general, a small proportion of the hit and false-alarm responses violate the average decision rule, and this causes the two performance indices to diverge by a small amount. The violation rate provides a slightly better description of the stability of the average criterion.

6. The subjects' use of close-to-neutral criterion values might have been rewarded by the trial-by-trial feedback. This feedback maximized the overall percent correct and, thus, encouraged adoption of neutral criteria. In addition, the net expected-gain function is relatively flat in the vicinity of ideal criterion, so that adoption of more extreme criteria would have increased the subjects' net gain by only a very small amount.

(Manuscript received June 10, 1992; revision accepted for publication July 20,1993 .) 